\title{
How age influences phonotaxis in virgin female Jamaican field crickets (Gryllus assimilis)
}

Female mating preference can be a dominant force shaping the evolution of sexual signals. However, females rarely have consistent mating preferences throughout their lives. Preference flexibility results from complex interactions of predation risk, social and sexual experience, and age. Because residual reproductive value should theoretically decline with age, older females should not be as choosy as younger females. We explored how age influences phonotaxis towards a standard mate attraction signal using a spherical treadmill (trackball) and a no-choice experimental protocol. Female Jamaican field crickets, Gryllus assimilis, were highly variable in their phonotaxis; age explained up to 64\% of this variation. Females 10 days post imaginal eclosion and older oriented toward the mate attraction signal, with 10- and 13-day females exhibiting the greatest movement in the direction of the signal. Our study suggests 10- and 13-day old females would be most responsive when quantifying the preference landscape for G. assimilis sexual signals. 
How age influences phonotaxis in virgin female

2 Jamaican field crickets (Gryllus assimilis)

3

4

Karen Pacheco, Jeff W. Dawson, Michael Jutting, and Susan M. Bertram ${ }^{1}$

$+5$

Department of Biology

Carleton University

1125 Colonel By Drive

Ottawa, Ontario, Canada K1S 5B6

9

10

$11{ }^{1}$ Corresponding author: Department of Biology ${ }_{2}$ Carleton University, 1125 Colonel By Drive, 12 Ottawa, Ontario, Canada K1S 5B6; Sue.Bertram@carleton.ca

Ottawa, Ontario, Canada K1S5B6

.

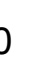




\section{ABSTRACT}

14 Female mating preference can be a dominant force shaping the evolution of sexual signals.

15 However, females rarely have consistent mating preferences throughout their lives.

16 Preference flexibility results from complex interactions of predation risk, social and sexual +17 experience, and age. Because residual reproductive value should theoretically decline with 18 age, older females should not be as choosy as younger females. We explored how age 19 influences phonotaxis towards a standard mate attraction signal using a spherical treadmill 20 (trackball) and a no-choice experimental protocol. Female Jamaican field crickets, Gryllus

21 assimilis, were highly variable in their phonotaxis; age explained up to $64 \%$ of this variation.

22 Females 10 days post imaginal eclosion and older oriented toward the mate attraction signal,

23 with 10- and 13-day females exhibiting the greatest movement in the direction of the signal.

24 Our study suggests 10- and 13-day old females would be most responsive when quantifying 25 the preference landscape for $G$. assimilis sexual signals. 


\section{INTRODUCTION}

Mate choice is a dominant force shaping the evolution of sexual signals (Andersson, 1994). Substantial research has focused on identifying which signals are preferred and how preferences maintain signal variation (Jacot et al., 2007; Jennions \& Petrie, 1997; Ritchie, 1996; Verburgt, 2008). Historically, many researchers assumed individuals make consistent mating preferences throughout their lives, resulting in relatively invariant selection influencing sexual signals (Andersson, 1994). However, individuals often exhibit flexible mating preferences (Kodric-Brown \& Nicoletto, 2001; Moore \& Moore, 2001; Lynch et al., 2005). Preference flexibility may result from complex interactions of many intrinsic and extrinsic factors including predation risk (Magnhagen, 1991; Godin, 1995), competition for available mates (Fawcett \& Johnstone, 2003), parasite load (Lopez, 1999), social and sexual experience (Dugatkin, 1992; Milinski \& Bakker, 1992; White \& Galef, 2000; Collins, 1995; Marler et al., 1997; Kasumovic et al, 2012), genetic makeup (Bakker \& Pomiankowski, 1995), and age (Jouventin et al., 1999; Qvarnstrom et al., 2000; Kodric-Brown \& Nicoletto, 2001; Moore \& Moore, 2001; Coleman et al., 2004; Lynch et al., 2005, 2006). Theoretically, residual reproductive value should decline with age (Roff, 1992; Stearns, 1992). Older females (with low residual reproductive value) may not be as choosy as younger females (with high residual reproductive value), because the benefits gained from being choosy may be offset by the increased costs of maintaining the preference (Real, 1980; Parker, 1983; Milinski \& Baker, 1992; Jennions \& Petrie, 1997). Costs associated with being choosy include time and energy in sampling, predation risk, and territorial quality (Jennions \& Petrie, 1997). Moore \& Moore (2001) 
demonstrated this in cockroaches (Nauphoeta cinerea). They found that older females were less choosy than younger females and the cost of being choosy (taking longer to select a mate) increased rapidly beyond an optimal age. Females past this optimal age required less courtship to mate and produced fewer offspring (Moore \& Moore, 2001).

Our study focuses on how age influences female field cricket phonotaxis. Male crickets defend territories from rivals and use these territories to broadcast acoustic mate attraction signals (Alexander, 1962). Males signal acoustically by rubbing their forewings together. Each closing stroke produces a pulse of sound; multiple pulses are concatenated into chirps (Bennet-Clark, 2003). Females exhibit phonotaxis towards male acoustic mate attraction signals and select between potential mates based on variation in male body size, mate attraction signals, courtship signals, aggressive behavior, and contact pheromones (Leonard \& Hedrick, 2009; Rebar et al., 2009; Thomas \& Simmons, 2010; Bailey, 2011; Beckers \& Wagner, 2011; Verburgt et al., 2011; Deb et al., 2012; Hedrick \& Kortet, 2012; Stoffer \& Walker, 2012).

Prosser (1994) was the first to investigate how age influenced female cricket mating preferences. Prosser et al (1997) revealed that older females (Gryllus texensis; aged 25-28 days post imaginal eclosion) exhibited greater movement toward a signal than younger females (11-14 days post imaginal eclosion) in one-speaker trials. Interestingly, both younger and older females showed strong preferences in three-speaker trials. Given older females responded to both trial types, older females seemed more motivated to mate. Conversely, younger females appeared more selective, only exhibiting preferences in trials with multiple mate opportunities (Prosser 
71 et al., 1997). Prosser et al's (1997) study suggests age influences mating preferences and highlights the importance of exploring preference across a broad range of ages. Most age studies have incorporated mating experience into their experimental design (e.g., Kodric-Brown \& Nicoletto, 2001; Mautz \& Sakaluk, 2008). While it makes sense to combine age with mating experience because the two are often positively correlated (Forslund \& Part, 1995; Prosser et al., 1997; Moore \& Moore, 2001; Martin \& Hosken, 2002; Mack et al., 2003), teasing apart the effect of age becomes more difficult. Judge et al. (2010) used a fully factorial experimental design to explore the relative importance of age and mating experience in female G. pennsylvanicus and revealed that mating experience was more important than age in influencing mating preferences. They suggest that because females are likely to mate quickly in the wild, any virgin age effects should be short-lived in nature.

Here we quantify how age influences female phonotaxis in the Jamaican field cricket, G. assimilis. Despite Judge et al.'s (2010) findings on the importance of mating experience, we chose to study only virgins because we needed to determine the best age to quantify virgin female phonotaxis for a subsequent study. We assessed phonotaxis slightly beyond the natural range of ages within which females are likely to be reproductively active in the wild (1-28 days post imaginal molt; Zuk, 1987; Cade, 1979). We quantified phonotaxis because females acoustically orient toward male mate attraction signals prior to mating (Alexander, 1962; Loher \& Dambach, 1989). We used a no-choice protocol and monitored phonotaxis toward a standard male acoustic attraction signal. Each female was tested only once so that our results were not confounded by experience or habituation. We used a spherical treadmill (trackball) to 
94 quantify phonotaxis (Kramer spherical treadmill or locomotion compensator: Kramer, 95 1976; Weber et al, 1981, Doherty, 1985, Doherty \& Pires, 1987; trackball: Hedwig \&

96 Poulet, 2005; Hedwig, 2006). When mounted on the spherical treadmill, the cricket 97 could walk in any direction with little or no encumbrance. We quantified each female's 98 locomotion over a set period of time with respect to a standard mate attraction signal 99 that was broadcast through a nearby speaker. Based on life history theory, we predicted 100 older virgin crickets would show greater movement toward the standard signal than 101 younger females. Our findings provide a relative standard for the degree of virgin female 102 G. assimilis responsiveness at a variety of ages. They also reveal the most responsive 103 age range to use when quantifying the preference landscape for $G$. assimilis sexual 104 signals.

\section{METHODS}

Adult G. assimilis were collected at the Stengl "Lost Pines" Biological Station 108 (University of Texas at Austin), in Bastrop County, Texas, United States (N 30 $5^{\prime}$ $\left.14.046^{\prime \prime}, W 97^{\circ} 10^{\prime} 28.988^{\prime \prime}\right)$ from 15-24 September 2008 and transferred to the 110 greenhouse laboratories at Carleton University, Ottawa, Ontario, Canada. Gryllus

111 assimilis were placed in communal plastic bins $(64 \times 40 \times 42 \mathrm{~cm})$ with a 16:8 h L:D

112 illumination period at $28 \pm 2{ }^{\circ} \mathrm{C}$ and fed ad libitum water [provided in individual plastic

113 containers ( $11 \mathrm{~cm}$ diameter $\times 3.5 \mathrm{~cm}$ height) filled with marbles to provide a surface for

114 the crickets to stand on] and food (Harlan's Teklad Rodent diet 8604M; 24.3\% protein, $11540.2 \%$ carbohydrate, $4.7 \%$ lipid, $16.4 \%$ fiber, 7.4\% ash; Harlan Laboratories, Madison, 116 WI, USA). Individuals mated freely and females were provided with moist sand 
117 [provided in individual plastic containers (11 cm diameter $\times 3.5 \mathrm{~cm}$ height)] in which to

118 lay their eggs. Juveniles developed in separate communal bins to minimize the

119 likelihood of larger crickets cannibalizing smaller crickets.

120 In 2012 we separated, 200 juvenile female crickets from the communal cricket

121 bins shortly after their ovipositor became visible. We housed these juveniles together

122 and monitored them daily for imaginal eclosion. Newly eclosed adult females were

123 housed individually in $520 \mathrm{~mL}(11 \mathrm{~cm}$ diameter $\times 7 \mathrm{~cm}$ height) clear plastic containers

124 with screened lids ( $4 \times 4 \mathrm{~cm}$ mesh screens). We provided each female with a small

125 piece of cardboard egg container for shelter and ad libitum water and food (as

126 described above); light cycles and temperatures were identical to the communal rearing

127 environment. We randomly assigned each female to an age group: 1-, 4-, 7-, 10-, 13-,

128 16-, 19-, 22-, 25- or 28-day post imaginal eclosion (hereafter day). We chose to use a

129 large number of age categories so we could quantify the age(s) at which the females

130 were exhibited the highest phonotaxis for a subsequent study on mating preferences.

131 Females were tested for their phonotaxis to a standard mate attraction signal on the day

132 they reached the age of their assigned age group.

133

Standard Mate Attraction Signal

135

We created a standard mate attraction signal (hereafter standard signal) using

Adobe Audition CS5.5 software. We fashioned this standard signal after results

137

published in Whattam and Bertram (2011). This study showed that male acoustic mate

attraction signaling is affected by diet; as such we selected parameters that reflect the

139

mean signal parameters from a population of males reared on high quality food (with the 
140 exception that Whattam and Bertram (2011) recorded their songs at $26^{\circ} \mathrm{C}$, while we 141 played these songs at $22-23^{\circ} \mathrm{C}$ ). The standard signal's parameters were: carrier

142 frequency $=3719 \mathrm{~Hz}$, pulse duration $=10.14 \mathrm{~ms}$, interpulse duration $=15.21 \mathrm{~ms}$, pulses

143 per chirp $=8$, interchirp duration $=1055 \mathrm{~ms}$; broadcast at an intensity of $60.6 \mathrm{~dB} \mathrm{SPL}$

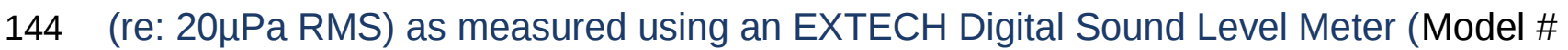

145 407768) with probe pointing directly at the active speaker, directly above the spherical

146 treadmill, $17 \mathrm{~cm}$ from the active speaker (Figure 1).

147

148 Spherical Treadmill Construction and Calibration

149 The inspiration for creating the spherical treadmill for quantifying cricket

150 locomotor behavior came from the earlier development of the "Kramer Treadmill"

151 (Kramer, 1976) and its extensive use by Huber and colleagues in their seminal studies

152 of the neural basis of cricket phonotaxis (Weber et al., 1981; Thorson et al., 1982;

153 Huber et al., 1984). This approach has seen various degrees of refinement and

154 re-invention as technologies have improved and studies have evolved with different

155 needs (e.g. Mason et al., 2001 for tracking tachinid fly orientation with respect to cricket

156 calling song stimuli). The principle idea of a Kramer treadmill is that it allows the cricket

157 to travel in different directions, at different velocities while maintaining constant distance

158 to a speaker.

159 Our spherical treadmill (Figure 2) is comprised of four principle components: 1. a

160 very-lightweight polystyrene ball that floats on a cushion of air, 2. a cowling that

161 constrains the ball and maintains the air cushion around the ball, 3. an electronic sensor 
162 that detects rotation of the ball, and 4. software that records the sensor readings and 163 calculates useful measures of the cricket's behaviour.

164 We chose the polystyrene ball based on its size (12.7 cm diameter) and small 165 mass (11.7 g) which is c. $5 \mathrm{X}$ larger than a typical female cricket walking on its surface. 166 The cowling (Figure 2B,C) was designed using CAD software then rapid-prototyped in 167 ABS plastic. The dimensions of the cowling were chosen after the polystyrene ball was 168 selected to ensure that the cowling had a small, c. $2 \mathrm{~mm}$, gap between itself and the ball. 169 Four holes were drilled through the cowling and outfitted with brass barbed hose fittings 170 (Figure 2D). Hoses attached to the fittings where then unified with a manifold and

171 connected to an $11 \mathrm{~L}$ oil-less air compressor to ensure equal air pressure at symmetric

172 locations beneath the ball. A collar was incorporated into the cowling design to prevent 173 the ball from being ejected by the air. The air stream cushion beneath the ball effectively

174 cancels the mass of the ball allowing the cricket to easily rotate the ball in multiple

175 directions. The polystyrene ball is not uniform in density and had a tendency to 'roll' in

176 one direction (without a cricket on its surface). To compensate for this, metal

177 dress-makers pins were inserted into the ball until flush with the surface at appropriate

178 locations to correct the centre of mass and thus counter-act these rotation motions

179 (Figure 2B).

To detect the motion of the ball, we extracted the sensor assembly, complete with

181 USB cable, from a generic optical mouse. This sensor assembly is comprised of a pair 182 of circuit boards with a complementary metal-oxide semiconductor (CMOS) camera and 183 a clear plastic lens. We fixed the circuit board with the CMOS camera and lens to a 184 window cut in the bottom of the cowling (Figure 2D). The distance of the camera and 
185 lens from the surface of the polystyrene ball is critical for the proper function of the 186 treadmill. We could adjust this distance with the screws used to attach the sensor 187 assembly to the cowling.

188 We wrote custom software using MATLAB $®$ (ver. 7.5, MathWorks, Natick MA, 189 USA) to detect the movement of the ball. Because the treadmill is essentially a 190 functioning USB optical mouse, we simply used standard functions in MATLAB ${ }^{\circledR}$ for 191 reading mouse pointer positions and sampled these data at a rate of $20 \mathrm{~Hz}$ during an 192 experiment trial. The change in mouse position reported by MATLAB ${ }^{\circledR}$ is in pixel units. 193 To convert between pixel units and $\mathrm{mm}$, we simply applied a thin strip of label tape 194 marked in mm increments to the ball surface and rotated the ball known distances in $\mathrm{X}$ 195 and $\mathrm{Y}$ directions. This allowed us to determine a conversion factor from pixel units to $196 \mathrm{~mm}$ of displacement. These control/calibration experiments also confirmed that the 197 rotation of the ball produced linear increments in $X$ and $Y$ directions. Note, the 198 calibration tape was removed for behavioural testing.

199

200

Spherical Treadmill Trials

201

We ran all experimental trials in the dark under red light in a chamber $(86 \times 57 \times$

$20287 \mathrm{~cm}$ ) lined with sound-attenuating foam. The spherical treadmill was placed in the 203 middle of the chamber with two speakers $17 \mathrm{~cm}$ from the center of the sphere and $180^{\circ}$ 204 from each other. The speakers and the spherical treadmill were always set in the same 205 location in the chamber.

206 We attached a coil- (micro-compression) spring (diameter: $3 \mathrm{~mm}$, length: $8 \mathrm{~mm}$;

207 spring constant: $210.15 \mathrm{~N} / \mathrm{m}$ ) to the cricket's pronotum with low melting point wax on the 
208 day of the trial (Figure 2A). Following a 10-min acclimatization period, we mounted the 209 female on the spherical treadmill by attaching her spring to a magnetic rod (Figure 2B).

210 The spring and magnet facilitated rapid changing of specimens. Air pressure was

211 adjusted such that the polystyrene ball, with the cricket in position, was able to rotate

212 freely (without frictional contact with the cowling). Each female was able to freely rotate 213 the polystyrene ball in all directions as she walked. All females were oriented in the 214 same neutral position at the start of their trial: tethered on the spherical treadmill facing 215 straight ahead between the two speakers. The magnet effectively kept the cricket 216 oriented in the same direction. Following a 1 min silent acclimatization period, we 217 presented the standard signal through a randomly selected speaker (to control for side 218 bias). After $30 \mathrm{~s}$ of exposure to the standard signal, we recorded the female's 219 phonotaxis relative to the broadcasting speaker. The standard signal was presented 220 continuously throughout the $5 \mathrm{~min}$ trial, and the female's locomotor behavior was 221 recorded from the treadmill at a sample rate of 20 samples per second. The 5 minute 222 trial therefore yielded a total of 6000 samples of cricket $X, Y$ positions. We freed the 223 female from the spring and returned her to the colony at the end of the trial.

224 Temperature was held constant (between $22-23^{\circ} \mathrm{C}$ ) in the trial room and was monitored 225 with a Fisher Scientific Traceable Digital Thermometer. We also note that the 226 compressor driving the spherical treadmill was located in a different room than the trials 227 and the noise of the compressor was not audible to the crickets under test. We calculated instantaneous displacement $(\mathrm{cm})$ and velocity $(\mathrm{cm} / \mathrm{s})$ vectors from 229 230 the positional data (X, Y coordinates). Total path length was calculated as the sum of all vector lengths over the 6000 samples. Net vector score (after Huber et al., 1984) was 
231 calculated as the female's net movement toward or away from the standard signal

232 during the 5 min trial. Net vector score takes into account the female's direction (vector 233 angle) and the vector length of every recorded leg of movement:

toward the speaker $\left(0^{\circ}\right)$ had positive vector scores $\left[\cos \left(0^{\circ}\right)=1\right]$, females moving directly away from the speaker $\left(180^{\circ}\right)$ had negative vector scores $\left[\cos \left(180^{\circ}\right)=-1\right]$, and females moving perpendicular to the speaker $\left(90^{\circ}\right.$ or $\left.270^{\circ}\right)$ had vector scores of $0\left[\cos \left(90^{\circ}\right.\right.$ or

$\left.270^{\circ}\right)=0$ ]. By multiplying this value by each vector length, and summing over the trial duration, we quantified the female's relative attraction to the standard signal. A large positive score indicated that the female moved quickly toward the speaker, a small

242 positive score indicated the female moved slowly toward the speaker, and a large negative score indicated the female moved quickly away from the speaker (Huber et al., 244 1984).

Statistical Analysis

All data were analyzed using JMP® statistical software (ver 10.0.0, SAS Institute Inc., 100 SAS Campus Drive, Cary, North Carolina, USA). Total path length and average instantaneous velocity magnitude were Box-Cox transformed because they were not normally distributed. We used one-way ANOVAs to quantify whether age influenced

251 female response to acoustic stimuli. We used Tukey HSD tests to ascertain significant 252 differences among the age groups when ANOVA results were significant. We corrected 
253 for multiple tests ( $N=4)$ using Benjamini and Yekutieli's (2001) false discovery rate

254 (FDR B-Y $)$ method; our FDR B-Y corrected alpha was $\mathrm{P}<0.024$.

255

256 RESULTS

257 Female age significantly influenced phonotaxis, explaining up to $64 \%$ of the 258 variation among females (Table 1). Females 10- to 13-days post imaginal eclosion 259 travelled longer total distance, walked faster and had higher net vector scores than 260 females at most other ages. Age significantly influenced total path length (Table 1) and 261 post hoc comparisons revealed that 10-day females moved further on the spherical 262 treadmill than all age groups except 13-day females (Figure 3A). Age also influenced 263 average instantaneous velocity magnitude (Table 1). Post hoc comparisons revealed 264 that 1- to 16-day females moved significantly faster than 22- to 28-day females, with 10265 and 13-day females having slightly higher velocities than other females (Figure 3B). 266 Further, age significantly influenced net vector scores (Figure 3C; Table 1). Post hoc 267 comparisons revealed that females 1-, 4-, and 7-days had significantly lower net vector 268 scores than females 10 -days or older. While the velocity of females less than 19-days 269 post imaginal molt was relatively high (Figure 3B), 1- to 7-day females did not generally 270 orient toward the speaker (Figure 4). 10- to 16-day females both oriented toward the 271 speaker and maintained fairly high velocities. Older females (22- to 28-day) oriented 272 toward the speaker but at low velocities (Figure 4).

273

274 DISCUSSION 
In crickets, and other acoustic insects, phonotaxis plays an important role in

276 mating, with females exhibiting positive phonotaxis toward the mate attraction signals of

277 conspecific males (Prosser, 1994; Cade, 1979; Sakaluk, 1982; Solymar \& Cade, 1990;

278 Jacot et al., 2007; Jennions \& Petrie, 1997; Ritchie, 1996; Verburgt, 2011). We found

279 age influenced female $G$. assimilis phonotaxis. Age explained $35-64 \%$ of the variation in 280 phonotaxis behavior, with females 10-13 days post adult eclosion displaying the

281 greatest movement toward the standard mate attraction signal compared to younger or 282 older females. Our study suggests that when quantifying the preference landscape for 283 G. assimilis sexual signals, 10- and 13-day females are likely to be the most responsive. 284 Some may criticise the use of a treadmill apparatus when quantifying female 285 phonotaxis because the intensity of the stimulus does not change within a trial despite 286 the locomotion behavior of the female. This is a valid criticism, as females are unable to 287 triangulate and localize the sound source and may, therefore, not respond naturally. 288 Regardless, we still found a statistically significant effect of age on orientation response 289 and thus feel our results are a conservative estimate of age effects.

290 We had hypothesized that younger females would be more responsive than older 291 females given their high residual reproductive value. While 1- to 7-day females traveled 292 long distances and at high speeds, they did not orient toward the broadcast mate 293 attraction signals (Figure 4). Their high speed and distances traveled may signify higher 294 energy reserves (Prosser, 1994), but their random orientation suggests little 295 responsiveness towards the standard acoustic mate attraction signal. There are at least 296 two possible explanations for their inattention to the signal: stronger preferences and/or 297 lack of maturity. Young females may have not oriented to our standard signal because it 
298 was not attractive. Females often prefer signals with greater energetic content (Wagner

299 et al, 1995) and extreme parameter values (Ryan \& Keddy-Hector, 1992). Conversely,

300 young females may have not oriented to our standard signal because they were not

301 reproductively mature. Average age to initiate phonotaxis in Acheta domesticus is 5.4

302 days following imaginal molt, and females do not mate until they reached 6.9 days on

303 average (Prosser, 1994). Other cricket studies also demonstrate onset of phonotaxis at

304 4-8 days post-imaginal molt (Walikonis et al., 1991; Loher et al., 1992). Future research

305 should present young females with multiple call stimuli to determine if they are

306 reproductively immature or only responsive to signal parameters with extreme trait

307 values.

308 10- and 13-day females exhibited greatest movement toward the standard signal.

309 Older females also oriented toward the standard signal. These findings partially support

310 our prediction that older females would show increased movement toward the standard

311 signal compared to younger females. However, older females tended to travel at much

312 slower speeds relative to younger females. Reductions in speed in older females may

313 indicate senescence (Prosser et al., 1997). Overall, our findings are consistent with

314 most studies on how age influences mating preference as we observed diminished

315 responsiveness with increasing age (Roff, 1992; Stearns, 1992; Gray, 1999; Wagner,

316 2004; Mautz \& Sakaluk, 2008).

317 While our study suggests that age is an important indicator of phonotaxis, it is

318 important to note that older females are unlikely to be virgin in the wild (Sakaluk, 1982;

319 Judge et al., 2010; Bretman \& Tregenza, 2005). Judge et al. (2010) revealed that

320 mating experience is more important than age in explaining female mating behavior. 
321 Judge et al (2010) used a fully factorial design where mated and unmated females of

322 two different age classes were scored for their latency to mate with random males

323 (no-choice mating paradigm). Given neither of our studies provided females with

324 alternative acoustic stimuli, future research should use multiple acoustic stimuli to

325 explore the relative importance of age versus mating experience on female preference

326 functions.

327

328 ACKNOWLEDGEMENTS

329 We would like to thank two anonymous reviewers for their comments on an earlier

330 version. We would also like to thank Ryan Chlebak for designing and digitally rendering

331 the spherical treadmill cowling and Andrew Mikhail who completed preliminary tests of

332 the device. Finally, we would like to thank Sarah J Harrison, Genevieve L Ferguson, Ian

333 R Thomson, and Kathryn Dufour for their help with cricket care. 


\section{REFERENCES}

335 Alexander RD. 1962. Evolutionary change in cricket acoustical communication. $336 \quad$ Evolution 16:443-467.

337 Andersson M. 1994. Sexual Selection. Princeton: Princeton University Press.

338 Bakker TCM, Pomiankowski A. 1995. The genetic basis of female mate preferences. $339 \quad$ Journal of Evolutionary Biology. 8:129-171.

340 Bailey RI, Innocenti P, Morrow EH, Friberg U, Qvarnström A. 2011. Female Drosophila 341 melanogaster gene expression and mate choice: the $\mathrm{X}$ chromosome harbours $342 \quad$ candidate genes underlying sexual isolation. PLOS ONE 6(2): e17358.

343 Beckers OM, Wagner WE Jr. 2011. Mate sampling strategy in a field cricket: evidence 344 for a fixed threshold strategy with last chance option. Animal Behaviour 81:519-527.

346 Benjamini Y, Yekutieli D. 2001. The control of the false discovery rate in multiple testing 347 under dependency. Annals of Statistics 29:1165-1188.

Bennet-Clark HC. 2003. Wing resonances in the Australian field cricket Teleogryllus oceanicus. Journal of Experimental Biology 206:1479-1496.

Bretman AJ, Tregenza T. 2005. Measuring polyandry in wild populations: a case study

351 using promiscuous crickets. Molecular Ecology 14:2169-2179.

352 Cade WH. 1979. Age of first mating in field crickets, Gryllus integer (Orthoptera:

$353 \quad$ Gryllidae). Florida Entomologist 73:193-195.

354 Coleman SW, Patricelli GL, Borgia G. 2004. Variable female preferences drive complex 355 male displays. Nature 428:742-745. 
356 Collins SA. 1995. The effect of recent experience on female choice in zebra finches.

$357 \quad$ Animal Behaviour 49:479-486.

358 Deb RM, Bhattacharya M, Balakrishnan R. 2012. Females of a tree cricket prefer larger 359 males but not the lower frequency male calls that indicate large body size. Animal Behaviour 84:137-149.

361 Dugatkin LA. 1992. Sexual selection and imitation: females copy the mate choice of 362 others. American Naturalist 139:1384-1389.

Fawcett TW, Johnstone RA. 2003. Mate choice in the face of costly competition.

Doherty JA. 1985. Trade-off phenomena in calling song recognition and phonotaxis in the cricket, Gryllus bimaculatus (Orthoptera, Gryllidae). Journal of Comparative Physiology A 156:787-801.

Doherty JA, Pires A. 1987. A new microcomputer based method for measuring walking phonotaxis in field crickets (Gryllidae). Journal of Experimental Biology

371 Behavioral Ecology 14:771-779. reproduction, female age, nutritional condition, body size, and size-relative reproductive investment. Journal of Insect Behavior 12: 691-700. crickets. Proceedings of the National Academy of Science 97:14449-14454. 
378 Godin J-GJ. 1995. Predation risk and alternative mating tactics in male Trinidadian 379 guppies. Oecologia 103:224-229.

380 Hedrick AV, Kortet R. 2012. Sex differences in the repeatability of boldness over

381 metamorphosis. Behavioral Ecology and Sociobiology 66:407-412.

382 Hedwig B, Poulet JF. 2005. Mechanisms underlying phonotactic steering in the cricket

383 Gryllus bimaculatus revealed with a fast trackball system. Journal of Experimental 384 Biology 208:915-927.

385 Hedwig B. 2006. Pulses, patterns and paths: neurobiology of acoustic behaviour in $386 \quad$ crickets. Journal of Comparative Physiology A 192:677-689.

387 Huber F, Kleindienst H-U, Weber T, Thorson J. 1984. Auditory behavior of the cricket. III.

$388 \quad$ Tracking of male calling song by surgically and developmentally one-eared 389 females, and the curious role of the anterior tympanum. Journal of Comparative $390 \quad$ Physiology A 155:725-738.

391 Jacot A, Scheuber H, Brinkhof MWG. 2007. The effect of age on a sexually selected $392 \quad$ acoustic display. Ethology 113:615-620.

393 Jennions MD, Petrie M. 1997. Variation in mate choice and mating preferences: a 394 review of causes and consequences. Biological Reviews 72:283-327.

395 Juventin P, LeQuette B, Dobson FS. 1999. Age-related mate choice in the wandering $396 \quad$ albatross. Animal Behaviour 57:1099-1106.

397 Judge KA, Tran KC, Gwynne DT. 2010. The relative effects of mating status and age on 398 the mating behaviour of female field crickets. Canadian Journal of Zoology $399 \quad 88: 219-223$ 
400 Johnsen TS, Zuk M. 1996. Repeatability of mate choice in female red jungle fowl.

$401 \quad$ Behavioral Ecology 7:243-246.

402 Kasumovic MM, Hall MD, Brooks RC. 2012. The juvenile social environment introduces

403 variation in the choice and expression of sexually selected traits. Ecology and

$404 \quad$ Evolution 2:1036-1047.

405 Kodric-Brown A, Nicoletto PF. 2001. Age and experience affect female choice in the 406 guppy (Poecilia reticulata). American Naturalist 157: 316-323.

407 Kramer E. 1976. The orientation of walking honeybees in odour fields with small $408 \quad$ concentration gradients. Physiological Entomology 1:27-37.

409 Leonard AS, Hedrick AV. 2009. Single versus multiple cues in mate discrimination by $410 \quad$ males and females. Animal Behaviour 77:151-159.

411 Lopez S. 1999. Parasitized female guppies do not prefer showy males. Animal $412 \quad$ Behaviour 57:1129-1134.

413 Loher WT, Weber T, Rembold H, Huber F. 1992. Persistence of phonotaxis in females of 414 four species of crickets following allatectomy. Journal of Comparative Physiology $A$ $415 \quad 171: 325-341$.

416 Loher W, Dambach M. 1989. Reproductive behavior. In: Huber F, Moore TE, Loher W, 417 ed. Cricket Behavior and Neurobiology. Ithaca, New York: Cornell University Press. $418 \quad 43-82$.

419 Lynch KS, Rand AS, Ryan MJ, Wilczynski W. 2005. Plasticity in female mate choice 420 associated with changing reproductive states. Animal Behaviour 69: 689-699. 
421 Lynch KS, Rand AS, Ryan MJ, Wilczynski W. 2006. Hormonal state influences aspects 422 of female mate choice in the Tungara Frog (Physalaemus pustulosus). Hormones $423 \quad$ and Behavior 49:450-457.

424 Mautz BS, Sakaluk SK. 2008. The effect of age and previous mating experience on pre425 and post-copulatory mate choice in female house crickets (Acheta 426 domesticus L). Journal of Insect Behavior 21:203-212.

427 Marler CA, Foran C, Ryan MJ. 1997. The influence of experience on mating 428 preferences of the gynogenetic Amazon molly. Animal Behaviour 53:1035-1041. 429 Magnhagen C. 1991. Predation risk as a cost of reproduction. Trends in Ecology and $430 \quad$ Evolution 6:183-186.

431 Mack PD, Priest NK, Promislow DEL. 2003. Female age and sperm competition:

432 last-male precedence declines as female age increases. Proceedings of the Royal Society of London B 270:159-165.

434

435

Martin OY, Hosken DJ. 2002. Strategic ejaculation in the common dung fly Sepsis cynipsea. Animal Behavior 63:541-546.

436

Mason AC, Oshinsky ML, Hoy RR. 2001. Hyperacute directional hearing in a microscale 437 auditory system. Nature 410:686-690.

438

Milinski M, Bakker TCM. 1992. Costs influence sequential mate choice in sticklebacks, 439 Gasterosteus aculeatus. Proceedings of the Royal Society of London B

440 250:229-233.

441 Moore PJ, Moore AJ. 2001. Reproductive aging and mating: The ticking of the biological 442 clock in female cockroaches. Proceedings of the National Academy of Science 443 98:9171-9176. 
444 Parker GA. 1983. Mate quality and mating decisions. Bateson P, ed. Mate Choice., 445 Cambridge: Cambridge University Press, 141-166.

446 Prosser MR. 1994. Effect of age on female choice in the field cricket, Gryllus integer. $447 \quad$ MSc Thesis, Brock University.

448 Prosser MR, Murray AM, Cade WH. 1997. The influence of female age on phonotaxis 449 during single and multiple song presentations in the field cricket, Gryllus integer 450 (Orthoptera: Gryllidae). Journal of Insect Behavior 10:437-449.

451 Qvarnstrom A, Part T, Sheldon BC. 2000. Adaptive plasticity in mate preferences linked 452 to differences in reproductive effort. Nature 405:344-347.

453 Rebar D, Bailey NW, Zuk M. 2009. Courtship song's role during female mate choice in 454 the field cricket Teleogryllus oceanicus. Behavioral Ecology 20:1307-1314.

455 Ritchie MG. 1996. The shape of female mating preferences. Proceedings of the $456 \quad$ National Academy of Science 93:14628-14631.

457 Roff DA. 1992. The evolution of life histories: theory and analysis. London: Chapman $458 \quad$ and Hall.

459 Real LA. 1990. Search theory and mate choice. I. Models for single-sex discrimination. $460 \quad$ American Naturalist 136:376-404.

461 Ryan MJ, Keddy-Hector A. 1992. Directional patterns of female mate choice and the $462 \quad$ role of sensory biases. American Naturalist 139:s4-s35.

463 Sakaluk SK. 1982. Onset of phonotaxis and age at first mating in female house crickets, $464 \quad$ Acheta domesticus (Orthoptera: Gryllidae). Journal of the New York Entomological 465 Society XC:136-141. 
466 Solymar B, Cade WH. 1990. Age of first mating in field crickets, Gryllus integer

467 (Orthoptera: Gryllidae). Florida Entomologist 73:193-195.

468 Stearns SC. 1992. The evolution of life histories. Oxford University Press, Oxford.

469 Stoffer B, Walker SE. 2012. The use of multimodal communication in mate choice

470 decisions by female house crickets, Acheta domesticus. Animal Behaviour

$471 \quad$ 83:1131-1138.

472 Thomas ML, Simmons LW. 2010. Cuticular hydrocarbons influence female

473 attractiveness to males in the Australian field cricket, Teleogryllus oceanicus.

$474 \quad$ Journal of Evolutionary Biology 23:707-14.

475 Thorson J, Weber T, Huber F. 1982. Auditory behavior of the cricket. II. Simplicity of

476 calling-song recognition in Gryllus, and anomalous phonotaxis at abnormal carrier

477 frequencies. Journal of Comparative Physiology A 146:361-378.

478 Verburgt L, Ferguson JWH, Weber T. 2008. Phonotactic response of female crickets on 479 the Kramer treadmill: methodology, sensory and behavioural implications. Journal $480 \quad$ of Comparative Physiology A 194:79-96.

481 Verburgt LM, Ferreira M, Ferguson JWH. 2011. Male field cricket song reflects age, 482 allowing females to prefer young males. Animal Behaviour 81:19-29.

483 Walikonis R, Schoun D, Zacharias D, Henley J, Cobum P, Stout J. 1991. Attractiveness

484 of the male Acheta domesticus calling song to females. III. The relation of

485 age-correlated changes in syllable period recognition and phonotactic threshold to 486 juvenile hormone III biosynthesis. Journal of Comparative Physiology A

$487 \quad 169: 751-764$. 
488 Wagner WE, Murray A-M, Cade WH. 1995. Phenotypic variation in the mating

489 preferences of female field crickets, Gryllus integer. Animal Behaviour

$490 \quad 49: 1269-1281$.

491 Weber T, Thorson J, Huber F. 1981. Auditory behavior of the cricket. 1. Dynamics of

492 compensated walking and discrimination paradigms on the Kramer treadmill.

$493 \quad$ Journal of Comparative Physiology 141:215-232.

494 Whattam EM, Bertram SM. 2011. Effects of juvenile and adult condition on

495 long-distance call components in the Jamaican field cricket, Gryllus assimilis.

$496 \quad$ Animal Behaviour 81:135-144.

497 Widemo F. Sæther SA. 1999. Beauty is in the eye of the beholder: causes and

498 consequences of variation in mating preferences. Trends in Ecology and Evolution

$499 \quad 14: 26-31$.

500 White DJ. Galef BG. 2000. Culture in quail: social influences on mate choices of female $501 \quad$ Coturnix japonica. Animal Behaviour 59:975-979.

502 Zuk M. 1987. Age determination of adult field crickets: methodology and field 503 applications. Canadian Journal of Zoology 65:1564-1566. 


\section{Figure 1}

Acoustic analysis of the standard mate attraction signal used for this study

Oscillograms of a single pulse (top) and chirps (second from top) comprising the signal. A spectrogram (fourth from top) with time-aligned oscillogram of a complete chirp (third from top) shows the presence of slight harmonics in the signal. (B) FFT spectrum of a complete chirp showing the dominant frequency (highest peak) and harmonics (subordinate peaks to the right of the dominant). Harmonic content was c. $30 \mathrm{~dB}$ below the energy of the peak frequency. The signal was synthesized and stored as a 32 bit mono WAV file with sampling frequency of $31250 \mathrm{~Hz}$. Spectrum was a 2048 point FFT with a Hanning window applied to the input signal. See text for additional details. 


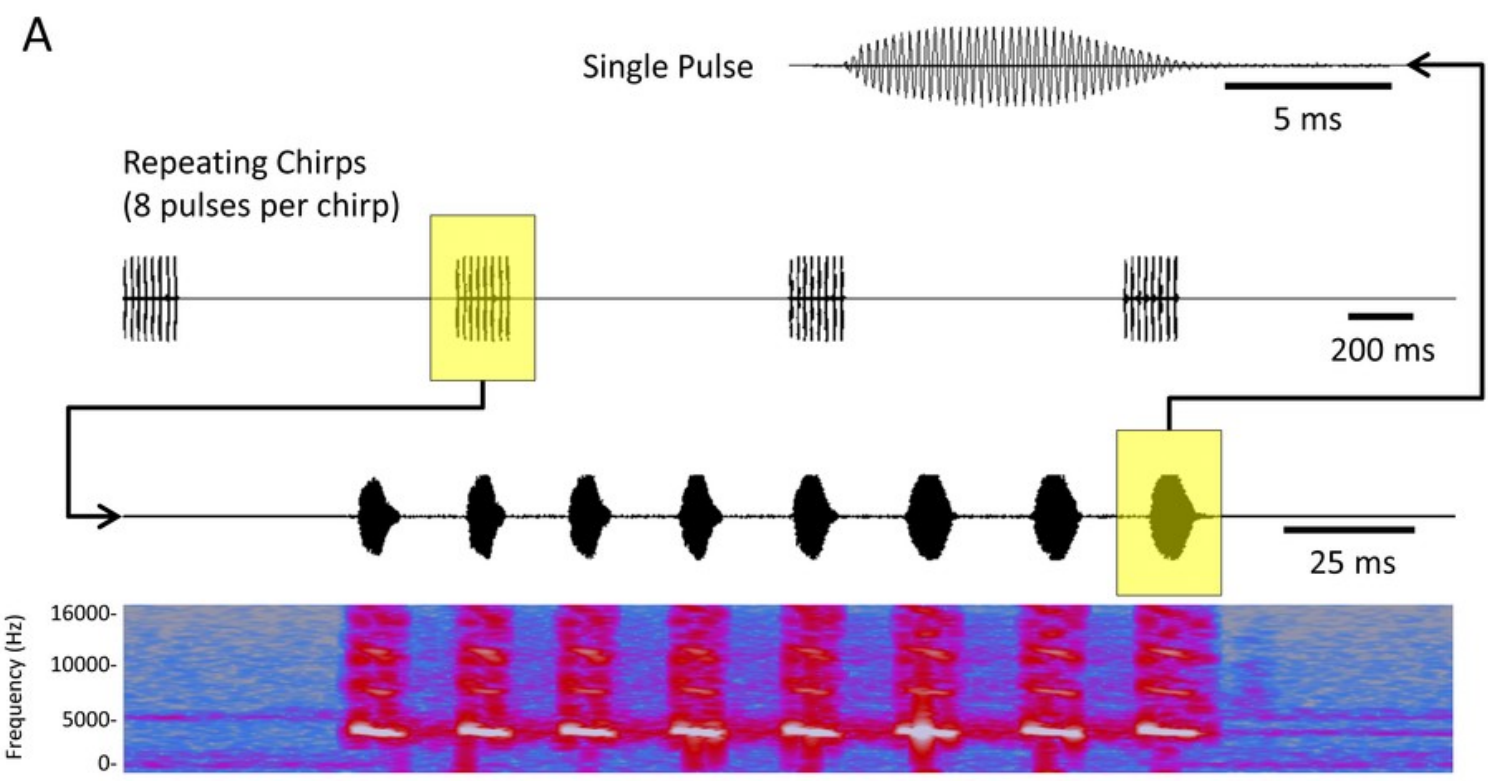

B

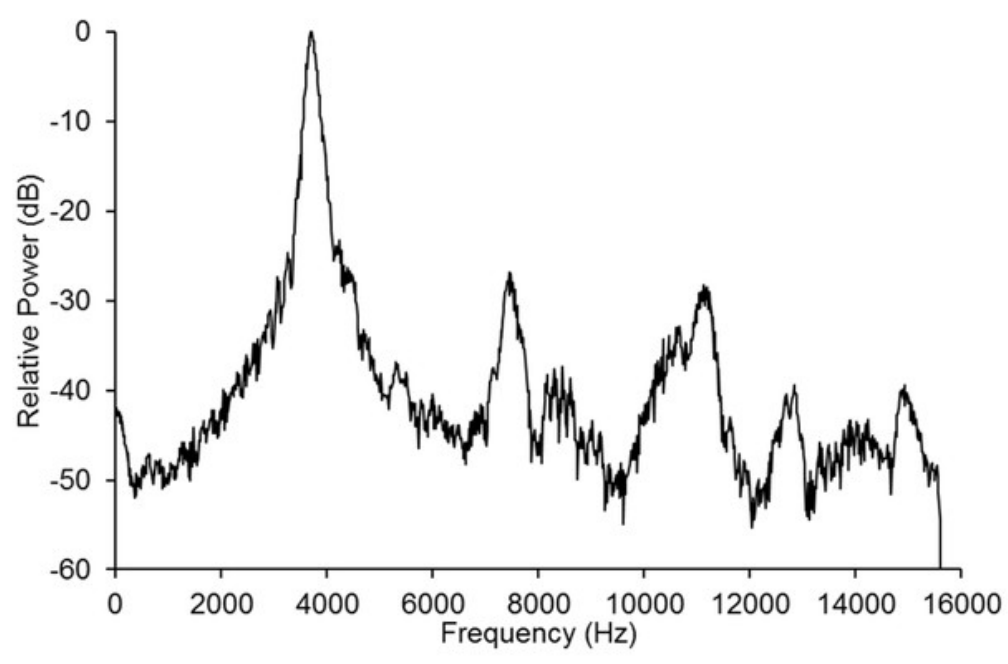




\section{Figure 2}

Photographs of the mounting spring and spherical treadmill apparatus.

(A) A female cricket (Gryllus assimilis) with coil spring attached to her pronotum with was. The spring is used to tether the cricket on the spherical treadmill while allowing her the freedom of body movements required for walking. (B) Female restrained on top of the spherical treadmill's polystyrene ball by her coil spring, magnet and rigid tether. Note the metal dress-makers pin flush with the surface of the polystyrene ball; then pins were used to counter-act rotation motions caused by the ball's non-uniform density. (C) The polystyrene ball was suspended on a cushion of air and was constrained by the trackball cowling and collar. Air hoses are not attached in this photograph. (D) A magnified view of the electronic sensor assembly including the CMOS camera and lens used to detect rotation of the ball. 


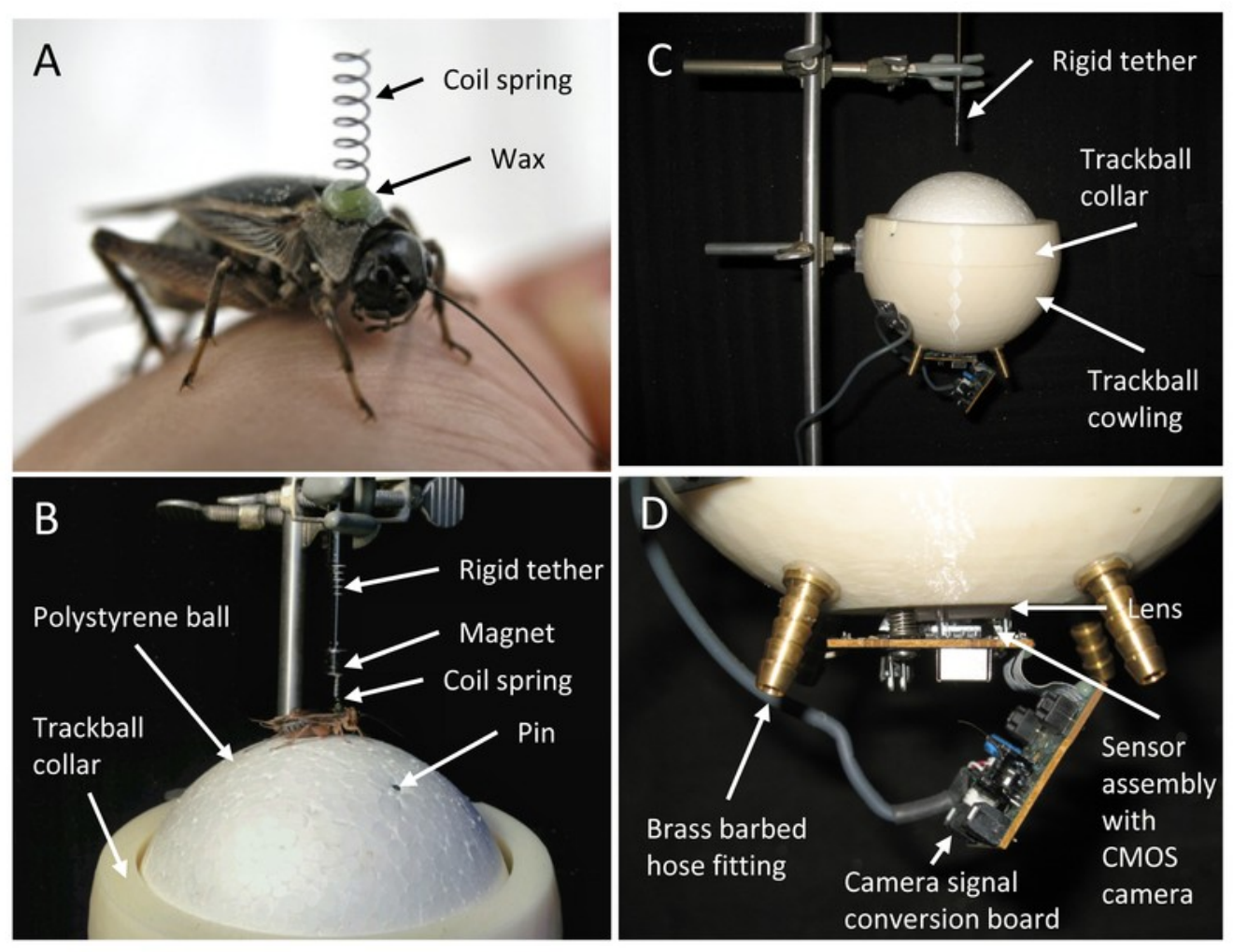




\section{Figure 3}

Box and whisker plots revealing how age influences female phonotaxis.

(A) toal path length (cm), (B) instantaneous velocity magnitude (cm/s), (C) net vector scores. Letters above each age show significant differences (Tukey HSD post-hoc analysis). 

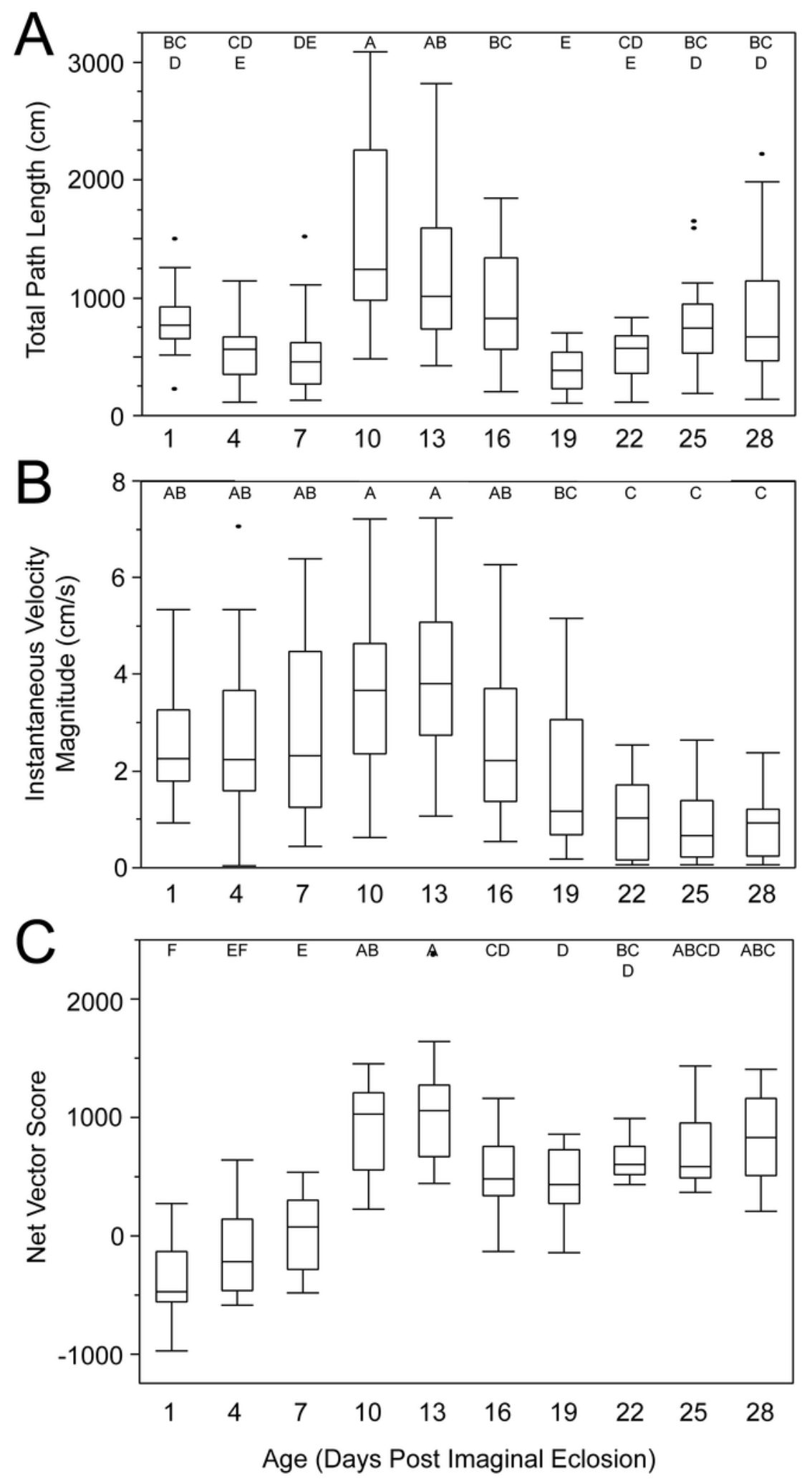


\section{Figure 4}

Polar plots of female velocity $(\mathrm{cm} / \mathrm{s})$ when presented with the standard acoustic mate attraction signal.

The speaker broadcasted the standard signal from the right (0 degrees); we converted the vectors for trials where the standard signal was broadcasted from the left so they appear on the right. Inner circular grid-lines represent velocity magnitude $(\mathrm{cm} / \mathrm{s})$; tic marks around the outside of the circle indicate velocity angle; the number in the upper left hand corner indicates female age (days post imaginal eclosion). Red arrows show the mean velocity for all 20 females at that age group. The larger, multi-colored plot at the bottom shows the differences in average velocity across all age groups. 

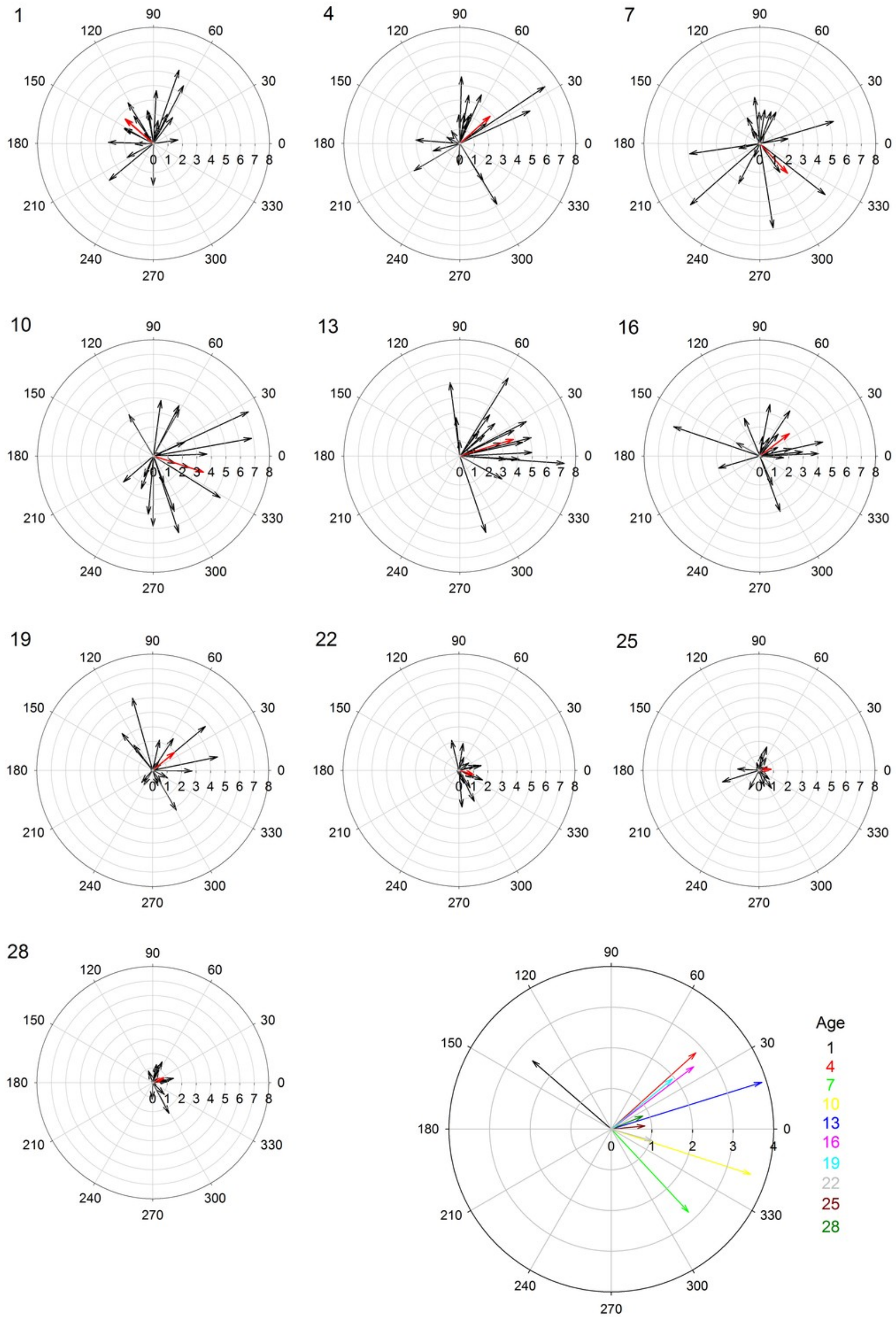


\section{Table $\mathbf{1}_{\text {(on next page) }}$}

ANOVA results showing the influence of age on phonotaxis measurements. 


\begin{tabular}{l|r|r|r|r}
\hline Phonotaxis measurement & DF & \multicolumn{1}{c|}{ F Ratio } & P value & \multicolumn{1}{c}{$\mathbf{R}^{2}{ }_{\text {adj }}$} \\
\hline Total Path Length (cm) & 1,9 & 12.9443 & $<0.0001$ & 0.3507 \\
Instantaneous Velocity Magnitude (cm/s) & 1,9 & 14.9834 & $<0.0001$ & 0.3874 \\
Net Vector Score & 1,9 & 40.2429 & $<0.0001$ & 0.6396 \\
\hline
\end{tabular}

